\title{
Incentives for input foreclosure
}

\author{
Roman Inderst ${ }^{\mathrm{a}, \mathrm{b}, *}$, Tommaso Valletti ${ }^{\mathrm{b}, \mathrm{c}, * *}$ \\ ${ }^{a}$ University of Frankfurt, Germany \\ ${ }^{\mathrm{b}}$ Imperial College London, UK \\ c Telecom ParisTech., France
}

\section{A R T I C L E I N F O}

\section{Article history:}

Received 17 November 2009

Accepted 20 December 2010

\section{JEL classification:}

L1

L4

Keywords:

Foreclosure

Vertical integration

Bilateral oligopoly

\begin{abstract}
A B S T R A C T
We analyze the incentives of a vertically integrated firm to foreclose downstream rivals in a model of upstream price competition between suppliers of only imperfectly substitutable inputs. Our main motivation is a critical assessment of common assertions that draw inferences from pre-merger observable variables to post-merger incentives to foreclose. In particular, we find that, contrary to some commonly expressed views, high margins on the downstream and low margins on the upstream market are not good predictors for the incentives of a newly integrated firm to foreclose rivals. Besides this contribution to policy, our model also extends existing results in the literature on vertical foreclosure through allowing for the interaction of product differentiation on the upstream and on the downstream market.
\end{abstract}

(c) 2010 Elsevier B.V. All rights reserved.

\section{Introduction}

This paper is motivated by a recent surge of interest in theories of competitive harm that arise from vertical integration. A key catalyst for this has been the revision of (non-horizontal) merger guidelines by several antitrust authorities, most notably the European Commission in late 2007. ${ }^{1}$ The European Commission suggests a three-step approach for assessing competitive harm from non-horizontal mergers: An appraisal of the ability to foreclose, of the incentives to foreclose and, finally, of the expected detriment to competition and consumers. This paper deals with the second step: the assessment of a newly vertically integrated firm's incentives to foreclose.

The EC's guidelines (EC 2007) develop several theories on when, based on pre-merger observables, incentives to foreclose should be higher or lower. ${ }^{2}$ They suggest, in particular, a relation between incentives to foreclose and pre-merger margins on the upstream and downstream markets. Other things constant, lower upstream margins and higher downstream margins should indicate higher incentives to foreclose (EC 2007, para 41). ${ }^{3}$ Though these theories are at first intuitive, our contribution

\footnotetext{
* Corresponding author. University of Frankfurt, IMFS, Grüneburgplatz 1, 60629 Frankfurt am Main, Germany.

** Corresponding author. Imperial College Business School, South Kensington Campus, London SW7 2AZ, UK.

E-mail addresses: inderst@finance.uni-frankfurt.de (R. Inderst), t.valletti@imperial.ac.uk (T. Valletti).

${ }^{1}$ Cf. also the guidelines issued by the Australian authority (ACCC) in 2008.

${ }^{2}$ It should be noted that the European Commission is much less reluctant than its US counterparts to pursue cases even when the fully or partially foreclosed input is not essential (e.g., a "bottleneck"), i.e., when the main theory of competitive harm is one of "raising rivals' costs".

${ }^{3}$ These theories were already applied earlier, e.g., to investigate a complicated joint venture and acquisition transaction in the European aerospace industry: Thales/Finmeccanica/Alcatel Alenia Space \& Telespazio COMP/M.4403. In its inquiry, the "[c]ommission considered some vertical arithmetic submitted by the parties in which the profitability of a foreclosure strategy was assessed; in line with the methodology outlined in the draft guidelines, using estimates of the mark-up upstream, the mark-up downstream, and the relative values of the input upstream and the output downstream, the diversion ratio downstream that would make the strategy of raising rivals' cost profitable was calibrated" (Neven and Albaek, 2007). Data on margins to document (the lack of) incentives to foreclose were also used recently in Philips/Intermagnetics COMP/M.4300.
} 
shows, instead, that they may also be misguided, as the policy maker should always need to ask first why pre-merger margins are high or low in the first place.

We show first how pre-merger margins may be high upstream but low downstream as downstream competition is intense, for instance as products are less differentiated. Still, we show how a newly integrated firm can then have higher incentives to fully foreclose downstream rivals than when downstream competition was less intense, therefore contrary to a casual application of the EC guidelines. This is because, when the downstream market is more competitive, the benefits of a cost advantage for the integrated firm compared to its non-integrated rivals are relatively larger than when competition is less intense. At the same time, with more intense competition, a variation in the input price for downstream rivals is also passedthrough to their final retail price to a larger extent than with less competition. Both these effects increase the benefits from foreclosure: the integrated firm, by not supplying input to its rivals, manages to increase their final price and get a substantial competitive advantage in the retail market. Then, foreclosure can be more likely to arise for strategic motives when the downstream market is already fairly competitive to start with, hence starting from a situation with ex ante observed low downstream margins.

These marginal effects are all ignored when looking only at the level of pre-merger up- and downstream margins, holding all else constant. In fact, our analysis makes clear that such a view of "holding all else constant" could be largely misleading: When upstream margins are high and downstream margins are low because of more intense downstream competition, then in the most basic case with linear demand this is associated with higher instead of lower incentives to foreclose.

We extend our analysis to the case where pre-merger margins differ as we vary competition in the upstream market or as we vary upstream costs of production. Again, our thereby obtained results reinforce the message that pre-merger margins alone, without additional information on characteristics of the up- and downstream markets, may provide little information about post-merger incentives to foreclose.

We should emphasize, however, that we do not claim to obtain always a prediction opposite to what may be suggested by a casual reading of the EC guidelines. A case where high pre-merger upstream margins may indeed indicate less incentives to foreclose is the following. When varying the degree of upstream competition, less competition increases pre-merger upstream margins, but it decreases pre-merger downstream margins, as input prices are higher. We illustrate how in this case, where we vary the degree of upstream competition, less competition and thus higher upstream and lower downstream margins make foreclosure less likely to arise in equilibrium.

Taken together, our main message is thus that one needs to go deeper and make a serious attempt to link pre-merger margins and foreclosure incentives to market primitives, such as the number of firms at various levels of the vertical chain, the degree of differentiation of their products, and the intensity of competition both up- and downstream. Absent such an analysis, pre-mergers margins, as such, are not revealing much about the incentives to foreclose following a vertical merger.

The model that we apply for our analysis is, additionally, of separate methodological interest. Our approach is to model price and quantity determination in the upstream market through Bertrand competition. While the seminal contribution of Salinger (1988) posits upstream Cournot competition, we thus follow Ordover et al. (1990) in assuming upstream price competition. ${ }^{4}$ However, we allow firms to move simultaneously. ${ }^{5}$ The analysis of upstream competition becomes non-trivial even though firms move simultaneously, as we allow for differentiated and non-essential inputs.

To obtain explicit results, we suppose that downstream firms have linear-quadratic costs, which are minimized by procuring a particular mix of inputs, depending on input prices. As one input becomes more costly, e.g., that of the newly integrated firm, then this affects both total and marginal costs of downstream production. This specification still allows for a fairly rich analysis, where we can vary, in particular, the degree of differentiation on both the downstream and the upstream market. Contrasting also downstream Cournot and Bertrand competition, as well as allowing for exogenous differences in efficiencies or (pre-merger) firm size, the model thus allows to obtain a rich set of predictions that relate changes in the incentives to foreclose to differences in pre-merger observables, such as market shares and margins.

A side insight from our analysis, which reinforces observations in the earlier literature, is that the presumption of upstream quantity competition, as in Salinger (1988), implies higher incentives to foreclose than with upstream price competition. ${ }^{6}$ The reason is the following. With price competition, when an integrated firm ends up supplying also its downstream rivals, the quantity that it sells partially replaces quantity that would otherwise be sold by rival suppliers. ${ }^{7}$ This is not the case with upstream Cournot competition, where the continuing supply of its downstream rivals has thus a larger effect on prices. Due to this replacement effect, there is less of a risk of foreclosure in case the upstream market is best described by price rather than quantity competition. In other words, results from extant literature on foreclosure that have found equilibrium foreclosure with upstream quantity competition, should be re-assessed as providing only an upper bound to the risk of foreclosure.

\footnotetext{
${ }^{4}$ See also Schrader and Martin (1998) for a critical view of using upstream Cournot competition. Church (2004) provides a detailed overview of the various models of foreclosure which typically assume that inputs are homogenous, an assumption we relax. A recent contribution that also introduces input differentiation is by Reisinger and Schnitzer (2009), though they are interested in the complementary question of endogenous market structure and do not analyze foreclosure incentives.

${ }^{5}$ Cf. the criticism in, for instance, Reiffen (1992) of the assumptions about the sequence of moves in Ordover et al. (1990).

${ }^{6}$ Cf. also the large follow-up literature using upstream quantity competition, such as Reiffen and Vita (1995) or Gilbert and Hastings (2005).

7 We do not consider the case of strategic overbuying the input, rather than selling it, to even further raise rivals'costs, though we discuss below when this may be profitable. Cf. also Gaudet and Van Long (1996) and Higgins (1999).
} 
Our focus on the assessment of theories on the use of pre-merger observables to predict foreclosure incentives is new and, as noted above, motivated by antitrust policy. To our knowledge, also our particular model of up- and downstream product differentiation is novel. ${ }^{8}$ As noted above, our paper follows a long tradition of modeling vertical relationships as markets, where non-integrated firms face a uniform price. A more recent literature takes, instead, a different perspective, focusing on bilateral contracts. ${ }^{9}$ One strand of this literature has followed Hart and Tirole (1990) in assuming that bilateral contracts are non-observable to third parties, which leads to a possibly severe problem of opportunism, such that the firm supplies all downstream firms at a marginal price that equals its own marginal cost. Given that a non-integrated supplier thus becomes himself his worst competitor, there is little scope to consider the impact of pre-merger upstream market structure on up-and downstream prices. From an applied policy perspective, this is potentially a serious drawback. ${ }^{10}$

Our analysis is concerned with firms' incentives to foreclose, and how these relate to pre-merger observables. Further, as a measure of foreclosure incentives we use whether post-merger a vertically integrated firm would optimally set its input price sufficiently high so as to choke off all demand from rival downstream firms. We then ask when this is more likely to be the case, depending on market characteristics that we finally link to pre-merger observables. We thus do not intend to provide a full analysis of the welfare implications of foreclosure. This is motivated also by practical interest, as we noted above that an analysis of foreclosure incentives often precedes a more complete analysis of its impact. When the integrated firm has an incentive to fully foreclose its rivals, then this pushes up input prices to the detriment of rivals. While traditionally economists are concerned only with the total welfare or the surplus of final consumers, current law and practice in Europe does not warrant this limitation, as it equally protects customers that are not final consumers. ${ }^{11}$

The fact that we are interested in providing guidance on integrated firms' incentives to foreclose, and how this relates to pre-merger observables, justifies also our interest only in incentives to fully foreclose downstream rivals. While this provides us with a clear and unambiguous way to capture foreclosure incentives, it must be noted that vertical integration and its impact on prices will have implications for welfare and consumer surplus even when there is no such full foreclosure. ${ }^{12}$

The rest of this paper is organized as follows. Section 2 introduces our model for the up- and downstream industries. Section 3 provides a short preview of the subsequent analysis. Section 4 then derives results for general downstream demand, with homogeneous goods. A detailed analysis with linear demand is contained in Section 5 . Section 6 concludes. All proofs are in the Appendix.

\section{The industry}

We consider a production process that takes place over two levels. At the first upstream level, there are $m \in M=\{1, \ldots, M\}$ different inputs. At the second downstream level, there are $n \in N=\{1, \ldots, N\}$ final goods. (Note that it is handy to use $M$ and $N$ both for the respective highest number as well as for the respective whole set.) In this section, we specify first the downstream production process.

We denote the quantity of input $m$ that goes into the production of good $n$ by $q_{n}^{m}$. Aggregate quantities are denoted by $q_{n}:=\sum_{m \in M} q_{n}^{m}$ and $q^{m}:=\sum_{n \in N} q_{n}^{m}$, respectively. Input $m$ is produced at constant marginal cost $c^{m}$. It is convenient to suppose that, for downstream firms, total output is just the sum of used inputs, $q_{n}$, so that we can dispense of an additional notation. All our results would hold, however, if we used a more general production technology with fixed proportions.

\subsection{Downstream costs of production}

The choice of inputs affects downstream costs of production. Suppose input $m$ is sold at the constant price $p^{m}$. When purchasing the quantity $q_{n}^{m}$ of input $m$, the total costs of production for good $n$ are given by

$$
\sum_{m \in M}\left[\delta\left(q_{n}^{m}\right)^{2} / 2+q_{n}^{m}\left(p^{m}+\beta\right)\right]
$$

\footnotetext{
${ }^{8}$ That being said, it is similar in spirit to the assumption of variable proportions as used in a vertical context by, for instance, Warren-Boulton (1974) or Quirmbach (1992). The focus of much of the more recent strand in the literature following Salinger (1988) and Ordover et al. (1990) is, however, different. In particular, newer contributions introduce upstream technology choice or costs of switching between suppliers, e.g., to enhance credibility of foreclosure (cf. Avenel and Barlet, 2000; Choi and Yi, 2000; Chen, 2001; Scheffman and Higgins, 2003 introduce a fixed cost of commitment). Normann (2009) studies raising-rivals'-costs strategies following a vertical merger using a repeated game.

9 While both approaches offer valuable insights, it may be argued that the assumption of a "market interface", with a uniform "merchant price" at the wholesale level, is potentially more realistic than bilateral contracts when several up- and downstream firms interact via short-term (spot) contracts and when there is little evidence of individually negotiated discounts (i.e., of wholesale price discrimination).

${ }^{10}$ See also Rey and Tirole (2007). When nonlinear bilateral contracts are, instead, publicly observable, then this can lead to a full monopolization of the downstream market, again regardless of the upstream market structure.

${ }^{11}$ According to the EC Commission, the concept of "consumer" includes all direct or indirect users of products covered by the practice under scrutiny, including producers that use the products as an input, wholesalers, retailers and final consumers (EC Commission Guidelines on the Application of Article 81(3) of the Treaty (2004) OJ C101/97). For a detailed discussion see Akman (2010). Such an objective that is wider than the enhancement of total welfare or that of final consumers is also shared by some national competition laws, most notably the German law.

12 Bourreau et al. (forthcoming) consider a model of two-tier competition between two vertically integrated firms and one unintegrated downstream firm. Because of a competition softening effect at the downstream level, they obtain partial foreclosure equilibria, in that the input may be priced above marginal cost in equilibrium. However, they rule out full foreclosure by assumption. See also Ordover and Shaffer (2007).
} 
In (1), $\beta \geq 0$ represents a constant marginal cost of transforming an additional unit of input into one unit of output. The parameter $\delta>0$ represents a measure of differentiation between inputs. Intuitively, the higher is $\delta$, the larger are the cost savings when spreading the total purchases more equally over different inputs. Instead, if $\delta$ is low, then inputs are close substitutes for the production of each downstream good. As is also noted in more detail below, the model is readily extended to include asymmetries between downstream products (i.e., in terms of different parameters $\beta_{n}$ and $\delta_{n}$ ).

Using (1), we have for each downstream product the costs of production

$$
C\left(q_{n}\right):=\min _{q_{n}^{m} \geq 0 \text { s.t. } \sum_{m \in M} q_{n}^{m}=q_{n}}\left\{\sum_{m=1, \ldots, M}\left[\delta\left(q_{n}^{m}\right)^{2} / 2+q_{n}^{m}\left(p^{m}+\beta\right)\right]\right\} .
$$

For positive output $q_{n}>0$, the minimization of the Lagrange function

$$
\sum_{m \in M}\left[\delta\left(q_{n}^{m}\right)^{2} / 2+q_{n}^{m}\left(p^{m}+\beta\right)\right]+\lambda\left(q_{n}-\sum_{m \in M} q_{n}^{m}\right)
$$

yields, in case of $q_{n}^{m}>0$, the first-order condition $\delta q_{n}^{m}+\beta+p^{m}-\lambda=0$. For any two inputs $m$ and $m^{\prime}$ where $q_{n}^{m}>0$ and $q_{n}^{m^{\prime}}>0$, we thus have that

$$
q_{n}^{m}-q_{n}^{m^{\prime}}=\frac{1}{\delta}\left(p^{m^{\prime}}-p^{m}\right) .
$$

Hence, a given price difference $p^{m^{\prime}}-p^{m}$ between two inputs $m^{\prime}$ and $m$ has, for given $q_{n}$, a larger effect on the difference in the respective purchases $q_{n}^{m^{\prime}}$ and $q_{n}^{m}$ if $\delta$ - our measure of differentiation in the upstream market - is low.

Lemma 1. Suppose that, so as to reduce total costs of production for given $q_{n}$, it is optimal to purchase positive quantities of all inputs, such that $q_{n}^{m}>0$ for all $m=1, \ldots, M$. Denoting with $\bar{p}_{\emptyset}:=\sum_{m \in M} p^{m} / M$, purchases of input $m$ equal

$$
q_{n}^{m}=\frac{q_{n}}{M}+\frac{\bar{p}_{\emptyset}-p^{m}}{\delta} .
$$

The resulting total costs of producing quantity $q_{n}$ are then

$$
C\left(q_{n}\right)=\sum_{m \in M}\left[\frac{\delta}{2}\left(\frac{q_{n}}{M}+\frac{\bar{p}_{\emptyset}-p^{m}}{\delta}\right)^{2}+\left(\frac{q_{n}}{M}+\frac{\bar{p}_{\emptyset}-p^{m}}{\delta}\right)\left(p^{m}+\beta\right)\right]
$$

and the respective marginal costs are

$$
C^{\prime}\left(q_{n}\right)=\delta \frac{q_{n}}{M}+\bar{p}_{\emptyset}+\beta
$$

Proof. See Appendix.

Note from (3) that it is indeed optimal to purchase from each input a positive quantity in case it holds for all $m \in M$ that

$$
p^{m}-\bar{p}_{\emptyset}<\delta \frac{q_{n}}{M} .
$$

Instead, when this does not hold for given $m$, then $q^{m}=0$. Furthermore, from the derivation in Lemma 1 it is immediate that, in case positive purchases are only made for a subset $M^{\prime} \subseteq M$ of inputs, then the respective marginal cost becomes instead, with a slight abuse of notation,

$$
C^{\prime}\left(q_{n}, M^{\prime}\right)=\delta \frac{q_{n}}{M^{\prime}}+\bar{p}_{\emptyset, M^{\prime}}+\beta,
$$

where $\left|M^{\prime}\right|>0$ is the size of the subset and $\bar{p}_{\emptyset, M^{\prime}}:=\sum_{m \in M^{\prime}} p^{m} / M^{\prime}$. This observation will be helpful once we turn to vertical integration and the potential of full foreclosure. Note, in particular, that when $M^{\prime}=M \backslash\{1\}$ and when, using the limiting condition from (6), $q_{n}$ is such that $p^{m}-\bar{p}_{\emptyset}=\delta q_{n} / M$, both total costs and marginal costs are the same, irrespective of whether firm $n$ had access to input $m$ or not: $C\left(q_{n}\right)=C\left(q_{n}, M^{\prime}\right)$ and $C^{\prime}\left(q_{n}\right)=C^{\prime}\left(q_{n}, M^{\prime}\right)$ when $p^{m}-\bar{p}_{\emptyset}=\delta q_{n} / M$ and therefore the downstream firm buys $M^{\prime}$ inputs only.

Finally, from (3) we also have that, provided all downstream products are produced using all inputs, the total quantity used of input $m$ is equal to

$$
q^{m}=\frac{q}{M}-\frac{N}{\delta}\left(p^{m}-\bar{p}_{\emptyset}\right),
$$

where $q=\sum_{m \in M} q^{m}=\sum_{n \in N} q_{n}$.

\subsection{Modified downstream production function}

With the presently chosen specification, if a given level of the total output $q$ is distributed over more downstream products $N$, then this reduces the total production costs. Essentially, in this case the introduction of more products represents also an increase in the number of downstream "plants", each operating with the same linear-quadratic cost function. To abstract 
from this effect, which would artificially affect the comparative statics in $N$, we want to keep the productive capacity in the final market constant when we change the number of products $N$. We next introduce such a modified set-up.

Suppose thus that there is, altogether, a productive capacity $K>0$ in the downstream market, with

$$
k:=K / N \text {. }
$$

This is owned symmetrically. Moreover, if quantity $q_{n}$ of some product is produced, production is optimally spread equally over these $k$ "plants". ${ }^{13}$ Each plant operates with the same cost structure, as given by (1). Total production costs for any given product are then $C_{k}\left(q_{n}\right)=k C\left(q_{n} / k\right)$. Note that in this case, if the total quantity $q$ is produced symmetrically in the downstream market, then total costs are now independent of $N: N C_{k}(q / N)=K C(q / K)$. Furthermore, with the technology $C_{k}\left(q_{n}\right)$ the marginal cost of production is from Lemma 1 equal to

$$
C_{k}^{\prime}\left(q_{n}\right)=\hat{\delta} N \frac{q_{n}}{M}+\bar{p}_{\emptyset}+\beta
$$

where we use

$$
\hat{\delta}:=\delta / K \text {. }
$$

In what follows, we work with (9). From our previous results in Lemma 1 we then have, in particular, that analogously to (8) it is

$$
q^{m}=\frac{q}{M}-\frac{1}{\hat{\delta}}\left(p^{m}-p_{\emptyset}^{u}\right)
$$

\section{Analysis: roadmap}

The general market game that we analyze is very simple. In the pre-merger case, at the first stage all suppliers choose their respective input prices. At the second stage, downstream firms compete in either prices or quantities. With price competition, they choose the respective prices and must subsequently meet the realized demand through purchasing the respective inputs. With quantity competition, buyers purchase inputs to produce a given output, which is then put on the market. With a vertical merger, the only difference is that a backwards integrated firm can purchase the respective input at marginal cost. Otherwise, the timing of moves is left unchanged.

Generally, we could allocate the $M$ different inputs and the $N$ different final goods to various up- and downstream firms, accommodating any asymmetric distribution of up- or downstream market power. However, for the purpose of the present analysis, which is mainly to provide some conceptual insights into the relationship between pre-merger observables and incentives to foreclose, we want to apply some specifications. We are, for obvious practical reasons, interested in the case where the integrating firms already have a strong position in their respective markets. We thus specify that the downstream firm that subsequently integrates backwards owns $1 \leq f \leq N-1$ products (including the respective "plants"). The remaining $N$ $-f$ firms operate independently. At the upstream level, the integrating firm subsequently controls input $m=1$.

Furthermore, to abbreviate terms we normalize the constant marginal cost of producing the potentially foreclosed input to $c^{1}=0$. With respect to the provision of other inputs, we specify that only a second input, $m=2$, is produced at marginal cost $c^{m}=\bar{c} \geq 0$. Moreover, this input is owned by more than one supplier. This allows us to heavily simplify the analysis, as we can thereby abstract from the strategic interaction of non-integrated input suppliers: They will always competitively provide the second input at the respective price $\bar{p}=\bar{c}$. Still, by having the parameter $\bar{c}$ at hands, we obtain in what follows variations in pre-merger margins that stem from variations in the upstream market. As noted in the Introduction, however, much of our insights will be obtained from varying characteristics of the downstream market. For this purpose, we now proceed as follows. To establish a baseline case, we first solve the case where downstream firms compete in quantities and sell a homogeneous good (Section 4). As is well known, this provides for a less competitive mode of competition than that of Bertrand competition, and our subsequent comparative analysis will also draw on this comparison. Furthermore, for the case of Bertrand competition, we will then provide in Section 5 a comparative analysis for a tractable model with linear demand.

\section{Homogeneous Cournot competition}

In this section, we specify that downstream products are undifferentiated. Firms compete downstream by choosing quantities $q_{n}$ and realize the common price $P(q)$.

\subsection{No vertical integration}

Note that without vertical integration and with homogeneous goods, firm $n=1$ only differs as it holds a larger "production capacity", namely of $k_{L}:=f K / N$ compared to $k=K / N$ for any of the $N-f$ firms. We denote the single quantity chosen by the first firm by $q_{1}$ and that of all other, smaller firms by $q_{n}$ for $n=2, \ldots, N-f+1$. Each one of the latter firms maximizes $q_{n} P(q)-C\left(q_{n}\right)$,

${ }^{13}$ More precisely, $k$ denotes here the measure of plants, of which each has infinitesimally small size. Literally speaking, we should then assure that $q_{n}>k$. By taking $K$ and thus $k$ to be sufficiently small throughout the analysis, $q_{n}>k$ would indeed always hold over the relevant output range. 
yielding the first-order condition ${ }^{14}$

$$
P(q)+q_{n} P^{\prime}(q)-C_{k}^{\prime}\left(q_{n}\right)=0,
$$

while the larger firm chooses $q_{1}$ to satisfy

$$
P(q)+q_{1} P^{\prime}(q)-C_{k_{L}}^{\prime}\left(q_{1}\right)=0 .
$$

Aggregating over (11) and (12), we have that

$$
(N-f+1) P(q)+q P^{\prime}(q)-(N-f) C_{k}^{\prime}\left(q_{n}\right)-C_{k_{L}}^{\prime}\left(q_{1}\right)=0 .
$$

Note next that from (9) due to Lemma 1 , we have $C_{k}^{\prime}\left(q_{n}\right)=\bar{p}_{\emptyset}+\beta+\hat{\delta}(N / M) q_{n}$ and $C_{k_{L}}^{\prime}\left(q_{1}\right)=\bar{p}_{\emptyset}+\beta+\hat{\delta}(N / M)(1 / f) q_{1}$, such that (13) transforms to

$$
(N-f+1) P(q)+q P^{\prime}(q)-(N-f+1)\left(\bar{p}_{\emptyset}+\beta\right)-(N-f) \hat{\delta} \frac{N}{M} q_{n}-\hat{\delta} \frac{N}{M} \frac{1}{f} q_{1}=0 .
$$

Together with $q=(N-f) q_{n}+q_{1}$, this yields

$$
\bar{p}_{\emptyset}=P(q)+\frac{1}{N-f+1}\left[q P^{\prime}(q)-(N-f) \hat{\delta} \frac{N}{M} q_{n}-\hat{\delta} \frac{N}{M} \frac{1}{f} q_{1}\right]-\beta .
$$

Expression (14) represents the indirect derived demand. This is obtained from indirect final demand, $P(q)$, after making the following adjustments. ${ }^{15}$ The term $q P^{\prime} /\left(N-f^{\prime}+1\right)<0$ captures the demand-reducing effect that arises from downstream firms' market power. This goes to zero as $N \rightarrow \infty$. Subtracting $\beta$ in (14) takes into account the marginal production cost that only depends on aggregate output. The terms with $\hat{\delta}$, instead, stem from the quadratic part of downstream production costs.

For the individual supplier, total demand for his input $m$ is obtained from aggregating individual demand over all buyers, $q_{n}^{m}$, i.e., $q_{n} / M-(1 / N \hat{\delta})\left(p^{m}-\bar{p}_{\emptyset}\right)$ for $n>1$ and $q_{1} / M-(f / N \hat{\delta})\left(p^{m}-\bar{p}_{\emptyset}\right)$ for $n=1$. This yields

$$
q^{m}=\frac{q}{M}-\frac{1}{\hat{\delta}}\left(p^{m}-\bar{p}_{\emptyset}\right) .
$$

Using our specifications of two inputs only, $M=2$, as well as the competitive provision of the second input at $\bar{p}=\bar{c}$, we have, in particular, that

$$
q^{1}=\frac{q}{2}+\frac{1}{2 \hat{\delta}}\left(\bar{c}-p^{1}\right) .
$$

At the first stage of the game, supplier $m=1$ chooses $p^{1}$ to maximize $q^{1} p^{1}$, where $q^{1}$ is obtained jointly from (14), which determines aggregate derived demand, and from (15), which determines the derived demand for the integrated firm.

\subsection{Vertical integration}

The difference in case of vertical integration is now twofold. First, firm $n=1$ can procure input $m=1$ at marginal cost, which is normalized to zero. Second, at the first stage of the game, $p^{1}$ is chosen to maximize the integrated firm's total profits over both the up- and the downstream markets. Hence, for $n=1$ we have the marginal cost of production

$$
C_{k_{L}}^{\prime}\left(q_{1}\right)=\bar{p}_{\emptyset}^{V I}+\beta+\hat{\delta} \frac{N}{M} \frac{1}{f} q_{1},
$$

where $\bar{p}_{\emptyset}^{V I}:=(1 / M)\left[c^{1}+\sum_{m^{\prime} \neq m} p^{m^{\prime}}\right]$ simplifies to $\bar{p}_{\emptyset}^{V I}=\bar{c} / 2$ in the special case with only two inputs. Note that with downstream quantity competition, the choice of $q_{1}$ does not affect the integrated firm's profits on the wholesale market, implying that $q_{1}$ still solves the first-order condition (12), albeit now with $\bar{p}_{\emptyset}^{V I}$ instead of $\bar{p}_{\emptyset}$. The first-order conditions for the non-integrated downstream firms $n>1$ are unchanged (as in (11)). Using the latter conditions, we obtain by aggregating over $n>1$ the derived demand for input $m=1$ from

$$
\bar{p}_{\emptyset}=P(q)+q_{n} P^{\prime}(q)-\hat{\delta} \frac{N}{M} q_{n}-\beta
$$

together with

$$
q^{1}=\frac{N-f}{2} q_{n}-\frac{N-f}{N} \frac{1}{\hat{\delta}}\left(p^{1}-\bar{p}_{\emptyset}\right)
$$

Expressions (16) and (17) mirror (14) and (15), respectively, for the case without vertical integration. The final requirement is that the choice of $q^{1}$ maximizes the total profits of the integrated firm: $\pi_{V I}:=q^{1} p^{1}+q_{1} P(q)-C_{k_{L}}\left(q_{1}\right)$.

\footnotetext{
${ }^{14}$ When not otherwise specified, we assume throughout this paper that the simple "first-order analysis" is justified: first, the respective optimal choice is interior; second, the underlying objective function is strictly quasiconcave; and, third, the equilibrium that results from the best-response functions is unique.

${ }^{15}$ See Inderst and Valletti (2009) for a more complete discussion of the relationship between direct demand in the final retail market and indirect demand in the intermediate wholesale market.
} 


\subsection{Incentives to foreclose}

The profit function $\pi_{V I}$ exhibits the standard trade-off between up- and downstream profits that the integrated firm faces when setting $p^{1}$. We ask under what circumstances the integrated firm would fully foreclose by setting a sufficiently high price that would choke off demand: $q^{1}=0$. As noted in the Introduction, this provides a comparable measurement for the incentives to foreclose. There, we also argued why a separate analysis of incentives is of key practical interests, even though it cannot be a substitute for a full analysis of welfare and consumer surplus.

Building on the assumed strict quasiconcavity of all objective functions, we proceed as follows. We first presume that $p^{1}$ is chosen sufficiently high such that $q^{1}=0$. At the prevailing equilibrium, and using then the level of $p^{1}$ that exactly chokes off demand, we calculate the profit impact for the vertically integrated firm when it marginally lowers $p^{1}$. We then characterize when foreclosure is indeed profitable, i.e., when $d \pi_{V I} /\left.d p^{1}\right|_{q^{1}=0}>0$.

This procedure allows to obtain the following result for general demand. There, we make use of $t:=d P(q) / d p^{1}$, which represents the pass-through rate of an input cost reduction (at $m=1$ ) for the $N-f$ independently owned firms.

Proposition 1. Suppose that there is downstream Cournot competition with homogeneous goods. As we approach the limit where inputs are perfect substitutes $(\delta \rightarrow 0)$ and if the pass-through rate satisfies $t<1 / 2$, then there is never full foreclosure, even when $\bar{c} \rightarrow c^{1}=0$, in which case the upstream margin goes to zero (while that of downstream firms is strictly positive).

\section{Proof. See Appendix.}

Before interpreting Proposition 1, it should first be noted that the restriction $t<1 / 2$ is not particularly strong. While it is well-known that there exist demand specifications (e.g., that of isoelastic demand) that have pass-through rates of common cost shocks that are larger than one, most (including linear demand) have pass-through rates that are strictly smaller. ${ }^{16}$ In addition, in our setting a marginal increase in $p^{1}$ increases marginal production costs by just half of it. Furthermore, note that $n=1$ is not affected by the increase in $p^{1}$ (i.e., $t$ is not the pass-through rate of a common cost shock for all firms). If quantities are strategic substitutes, which is the common specification, then this further reduces the pass-through rate $t$.

Proposition 1 comprises two results that are subsequently made more explicit and precise with linear demand. First, with upstream price competition instead of quantity competition as in much of the extant literature, incentives for (full) foreclosure so as to thereby raise rivals' costs may be rather small (cf. more precisely condition (A.5) in the proof contained in the Appendix). Second, there is no (full) foreclosure even when the ratio of down- to upstream margins goes to infinity as upstream products become increasingly close substitutes.

For some further discussion, we obtain for $\bar{c}=0$ the simplified condition that there is no (full) foreclosure whenever

$$
t\left|\frac{1}{P^{\prime}}\right|\left[P-C_{k_{L}}{ }^{\prime}\left(q_{1}\right)-\frac{p^{1}}{2}\right]<\frac{q_{n}}{2} .
$$

Note that, in contrast to Proposition 1, condition (18) holds not only in the limit where $\delta \rightarrow 0$. While, as argued above, $t<1 / 2$ in Proposition 1 was not much restrictive, condition (18) imposes a more general restriction. While only additional functional specifications would allow to make more precise statements, it is instructive to observe that condition (18) is more easily satisfied the lower is $t$. Intuitively, the lower is the pass-through rate, the less will a reduction of $p^{1}$ (and thus, in particular, less-than-full foreclosure) impact on downstream rivals' output. Typically, the pass-through rate is higher when the (downstream) market is more competitive, implying that condition (18) is then less likely to hold, such that full foreclosure may prevail. We make this insight more explicit once we assume linear demand, as done in the subsequent section.

\section{Linear demand}

In this section, we consider a case with linear demand and product differentiation. From the linear-quadratic specification of a representative consumer's utility that is used in Shubik and Levitan (1980), we obtain for good $n$ the demand function

$$
q_{n}=\frac{1}{N}\left[1-p_{n}-\theta\left(p_{n}-\frac{\sum_{n^{\prime} \in N} p_{n^{\prime}}}{N}\right)\right]
$$

and the indirect demand function

$$
p_{n}=1-\frac{N+\theta}{1+\theta} q_{n}-\frac{\theta}{1+\theta} \sum_{n^{\prime} \neq n} q_{n^{\prime}} .
$$

This demand system has the attractive property that, when changing the degree of substitutability $\theta$, total as well as individual quantities do not change in case firms charge symmetric prices. ${ }^{17}$ Products are independent if $\theta=0$ and become increasingly substitutable as $\theta$ increases. When $\theta \rightarrow \infty$ we get back to the case of homogeneous goods considered in the previous section.

\footnotetext{
16 See Weyl (2008) for a thorough discussion of pass-through rates.

17 Höffler and Schmidt (2008) use this formulation to investigate the related topic as to when a vertically integrated firm prefers to sell to independent resellers.
} 


\subsection{No foreclosure with downstream Cournot competition}

Though now downstream products are differentiated, the procedure is the same as for the general analysis with homogeneous products. We obtain the following stark result.

Proposition 2. With downstream Cournot competition and linear demand, there is never full foreclosure.

Proof. See Appendix.

As noted in the Introduction, that incentives to foreclose are thus relatively low in the present model follows from the fact that suppliers compete in prices. While with quantity competition the integrated firm's additionally supplied quantity on the merchant market increases the total quantity of all inputs by the same amount, with price competition there is a replacement effect. The impact on the downstream market is thus smaller. From this perspective, results from the extant literature on foreclosure that are obtained with upstream quantity competition should thus be viewed as providing at best an "upper bound" with respect to the incentives to foreclose.

\subsection{Potential for foreclosure with downstream Bertrand competition}

We limit our explicit analysis to the cases of very high and very low values of $\theta$, where products become very homogeneous or heterogeneous, respectively. Without much loss of generality, we presently set $\bar{c}=0$ to shorten expressions. (The main role of $\bar{c}$ is to conduct comparative statics exercises that we perform further below.) For the critical derivative $d \pi_{V I} /\left.d p^{1}\right|_{q^{1}}=0$ we obtain at the limit $\theta=0$, where products are undifferentiated, that

$$
-(N-f)(1-\beta) \frac{\hat{\delta}}{N \hat{\delta}(4+\hat{\delta})}<0,
$$

implying that there is no full foreclosure, both in the limit and - by appealing to continuity - also for sufficiently low $\theta$. At the other extreme, where $\theta \rightarrow \infty$, we obtain from the condition $d \pi_{V I} /\left.d p^{1}\right|_{q^{1}=0}<0$ that there is no full foreclosure if and only if

$$
-(1-\beta) \frac{f(4+\hat{\delta})-2 N(1+\hat{\delta})}{2 N\left(2+3 \hat{\delta}+\hat{\delta}^{2}\right)>0,}
$$

which in turn holds if and only if

$$
\frac{f}{N}<2 \frac{1+\hat{\delta}}{4+\hat{\delta}} .
$$

Appealing to continuity (in $\theta$ ), we thus have the following results.

Proposition 3. With linear demand and downstream Bertrand competition, there are no incentives to foreclose if goods are sufficiently differentiated. Instead, if goods are sufficiently close substitutes, then there is full foreclosure if and only if f/N is sufficiently large.

Condition (20) is intuitive: Incentives to foreclose are higher as the integrated firm controls more products and has thus a larger share of the downstream market, implying that it will benefit to a larger scale in case its rivals' costs and thus their final prices are increased. ${ }^{18}$

A corollary of Proposition 3, together with Proposition 2, is that when the mode of downstream interaction is more competitive, as in the case of Bertrand instead of Cournot competition, then the risk of foreclosure is higher. We argue now that this observation, together with the comparative analysis in Proposition 3, suggests that lower downstream and higher upstream margins are potentially indicative for higher incentives to foreclose, at least when the underlying comparative analysis is with respect to conditions on the downstream market.

\subsection{Incentives to foreclose: variation in the downstream market}

As the downstream market becomes more competitive, as either products become less differentiated (higher $\theta$ ) or as the mode of competition is Bertrand instead of Cournot, then the (pre-merger) margins of downstream firms are strictly lower. The pre-merger upstream margin for $m=1$ should, instead, be strictly higher. In both cases, however, incentives to foreclose are higher as well. We now provide a formal proof of these observations.

By appealing to standard insights for a comparison of Cournot competition with Bertrand competition - though this can be easily made explicit in the present setting - we have the following argument. All else constant, each input faces a larger demand under downstream price competition, making it indeed optimal to charge a higher price, which we denote by

18 This confirms another theory on the incentives to foreclose that has been formulated in the EC's new non-horizontal merger guidelines (European Commission 2007, para. 43) 
$p_{B}^{1}>p_{C}^{1} .^{19}$ The upstream margin is thus higher under downstream Bertrand competition. That the downstream margin is lower follows next immediately from combining the following two steps: First, for a given input price we know that the downstream margin would be smaller under Bertrand competition; second, even when adjusting equilibrium quantities, the margin further decreases from $p_{B}^{1}>p_{C}^{1}$.

Turning next to a comparative analysis in $\theta$, using Proposition 3 we again restrict ourselves to a comparison of the extreme cases where $\theta \rightarrow 0$ or $\theta \rightarrow \infty$. Altogether, we obtain the following results.

Proposition 4. As the downstream market becomes more competitive, this reduces pre-merger downstream margins and increases the pre-merger upstream margin, though incentives to foreclose are higher. This holds both for a switch from Cournot to Bertrand competition, as well as for the case when products become less differentiated (precisely, for $\theta \rightarrow 0$ vs. $\theta \rightarrow \infty$ ).

Proof. See Appendix.

It should be noted that the restriction to $\theta \rightarrow 0$ and $\theta \rightarrow \infty$ is only made to obtain relatively short expressions, as for general $\theta$ these become tedious. All numerical examples that we calculated reveal, however, that incentives to foreclose change monotonically in $\theta$ : That is, there is a strictly positive cutoff $\theta^{\prime}$ such that $q^{1}>0$ only when $\theta<\theta^{\prime}$.

If the exogenous change is with respect to the degree of downstream competition, then high pre-merger upstream and low pre-merger downstream margins are indicative of high - rather than low - incentives to foreclose, which is opposite to the EC's guidelines.

\subsection{Incentives to foreclose: variation in the upstream market}

Recall that input $m=2$ is supplied competitively, given that it is offered by more than one supplier. To conduct a comparative analysis in the upstream market, we now consider a change in $\bar{c}$.

It is intuitive that, following an increase in $\bar{c}$, the pre-merger upstream margin increases, while the pre-merger downstream margin decreases, given the enhanced pricing power of supplier $m=1$. Recall from the detailed exposition in the Introduction, that in case of a change in downstream competition there were two conflicting effects that impact on premerger margins and subsequent incentives to foreclose: On the one hand, by reducing downstream and increasing upstream margins, more competition reduces incentives to foreclose; but, on the other hand, more competition increases the marginal impact that participation has on downstream profits. When we consider, instead, a change in $\bar{c}$, there simply is no such countervailing effect. The following result is then immediate. ${ }^{20}$

Proposition 5. Suppose pre-merger margins change as $\bar{c}$ increases, enhancing the pricing power for supplier $m=1$. Then the relationship between incentives to foreclose and pre-merger margins is the opposite to that in Proposition 4.

Proposition 5 provides a clear-cut result, given that for this comparative analysis there is only one channel through which incentives to foreclose are affected: the level of up- and downstream margins. In contrast, when we changed downstream competition, this "level effect" had to be contrasted with the "marginal impact" effect, where the latter dominated with linear demand.

A more far reaching modification to the upstream market would be to change the mode of competition. While our model considers upstream Bertrand competition, we could also apply upstream Cournot competition, as in much of the literature that we surveyed in the Introduction. While this leads to higher pre-merger margins for $m=1$, incentives to subsequently foreclose downstream rivals may be smaller under upstream Cournot competition, as there is no "replacement effect": Continued participation has a higher impact on downstream prices. It can be shown that this effect may again be sufficiently strong to more than compensate for the "level effect", i.e., the higher upstream and the smaller downstream margins under upstream Cournot compared to upstream Bertrand competition.

Together with our previous results, this final observation highlights one of the core messages of the present paper: Information from pre-merger margins, when considered in isolation, can only be informative on incentives to foreclose if it is well understood why pre-merger margins are high or low in the first place.

\section{Concluding remarks}

This paper introduces a model that allows to study hypotheses that relate up- and downstream market characteristics and pre-merger observables to an integrated firm's incentives for input foreclosure. In light of recent policy discussions, one focus of our paper is on the predictive value of pre-merger up- and downstream margins.

\footnotetext{
${ }^{19}$ As this step is potentially the one that is least immediate, note that in the linear case one can also express derived demand as $q_{C}^{1}=a_{C}-b_{C} p^{1}$ for Cournot and as $q_{B}^{1}=a_{B}-b_{B} p^{1}$ for Bertrand. That $q_{B}^{1}>q_{C}^{1}$ follows immediately from the fact that for given $\left(\bar{c}, p^{1}\right)$ all final quantities $q_{n}$ are strictly higher (if positive) under Bertrand. To then obtain that in equilibrium $p^{1}$ will be strictly higher under Bertrand than under Cournot competition, it thus must hold that $a_{B} / b_{B}>a_{C} / b_{C}$, which follows as derived demand is strictly larger under Bertrand competition.

${ }^{20}$ A proof is omitted for brevity's sake, as the respective terms, for general $f$ and $\theta$, become very long. Importantly, after some reshuffling, one can show that in $d \pi_{v /} /\left.d p^{1}\right|_{q^{1}=0}$ the collected terms related to $\bar{c}$ have indeed a negative sign.
} 
We show that a focus on the size of these margins is insufficient to appropriately assess the incentives for subsequent input foreclosure. Instead, a firm's incentive to still sell on the merchant market is driven also by the price impact that this has on the downstream market (pass-through) and on the competitive advantage that a cost differential bestows on the integrated firm. As we show, this impact can be high precisely when the downstream margin is low and the upstream margin is high.

A second insight of the paper is obtained by contrasting changes in up- and downstream market characteristics. We showed that the relationship between pre-merger margins and foreclosure incentives is not robust but, instead, depends crucially on the reason for why margins are low or high in the first place, e.g., due to more or less competition on the upstream or on the downstream market.

The linear-quadratic model of input and retail price differentiation that we apply in our analysis mainly for illustrative purposes is also of interest in itself. As we noted above, though our analysis focuses for tractability mainly on the case of symmetric differentiation, the general set-up allows to introduce asymmetries at various points. Together with the simplicity of our set-up, this may make the model easily applicable for quick simulation exercises in merger analysis.

\section{Acknowledgments}

We thank an anonymous referee, the Associate Editor, Thibaud Vergé and seminar participant at CRESSE (Crete), the European Commission (Brussels), and the Office of Fair Trading (London). Support from ESRC Grant RES-062-23-2099 is gratefully acknowledged.

\section{Appendix A}

Proof of Lemma 1. Using (2) to sum up over all $M$, we have

$$
(M-1) q_{n}^{m}=\left(q_{n}-q_{n}^{m}\right)+\frac{\sum_{m^{\prime} \in M / m} p^{m^{\prime}}-(M-1) p^{m}}{\delta},
$$

which after transformation and division by $M$ yields (3). For further reference, it is also helpful to write this as

$$
q_{n}^{m}=\frac{q_{n}}{M}+\frac{1}{M} \frac{1}{\delta} \sum_{m^{\prime} \neq m} p^{m^{\prime}}-\frac{1}{\delta} \frac{M-1}{M} p^{m} .
$$

Substituting (3) into the total costs (1), we have (4), which after differentiation yields

$$
C^{\prime}\left(q_{n}\right)=\frac{1}{M} \sum_{m \in M}\left[\delta\left(\frac{q_{n}}{M}+\frac{\bar{p}_{\emptyset}-p^{m}}{\delta}\right)+\left(p^{m}+\beta\right)\right]
$$

and thus, after some further transformation, (5) is obtained.

Proof of Proposition 1. Note first that demand for $m=1$ is exactly zero if, using (17), it holds that

$$
p^{1}=\bar{c}+q_{n} N \hat{\delta} .
$$

We consider the derivative $d \pi_{V I} / d p^{1}$ at the level (A.2). If it is strictly negative, we can rule out full foreclosure. ${ }^{21}$ From the envelope theorem, when applied to the maximization of $q_{1} P(q)$, we have

$$
\left.\frac{d \pi_{V I}}{d p^{1}}\right|_{q^{1}=0}=p^{1} \frac{d q^{1}}{d p^{1}}+q_{1} P^{\prime}(q)(N-f) \frac{d q_{n}}{d p^{1}} .
$$

Substituting for $q^{1}$, next to $d q^{1} / d p^{1}=((N-f) / 4)\left[d q_{n} / d p^{1}-1 / N \hat{\delta}\right]$, and $p^{1}$ from (A.2), we obtain that $d \pi_{v_{I}} /\left.d p^{1}\right|_{q^{1}}=0<0$ holds if

$$
\left(\bar{c}+q_{n} N \hat{\delta}\right) \frac{1}{2}\left[\frac{d q_{n}}{d p^{1}}-\frac{1}{N \hat{\delta}}\right]+q_{1} P^{\prime}(q) \frac{d q_{n}}{d p^{1}}<0 .
$$

For a final transformation, we use that

$$
\frac{d q_{n}}{d p^{1}}=\frac{d P(q)}{d p^{1}} \frac{d q_{n}}{d P}=\frac{d P(q)}{d p^{1}} \frac{1}{P^{\prime}(q)}=\frac{t}{P^{\prime}} .
$$

(Recall that $t=d P(q) / d p^{1}$.) We then have from (A.4) that there is no full foreclosure if

$$
t q_{1}-\frac{q_{n}}{2}+q_{n} N \hat{\delta} \frac{t}{2 P^{\prime}}+\frac{\bar{c}}{2}\left[\frac{t}{P^{\prime}}-\frac{1}{N \hat{\delta}}\right]<0
$$

\footnotetext{
21 Though with a kink at (A.2) $\pi_{V I}$ is not continuously differentiable, for this analysis we only have to use the left-side derivative.
} 
and thus if

$$
t q_{1}<\frac{q_{n}}{2}+\frac{\bar{c}}{2 N \hat{\delta}}+\left|\frac{1}{P^{\prime}}\right| \frac{t}{2}\left(\bar{c}+q_{n} N \hat{\delta}\right) .
$$

Condition (A.5) represents a very strict condition. In fact, as we show below for the case with linear demand, it is never satisfied. ${ }^{22}$ As $\bar{c}=0$ and $\hat{\delta} \rightarrow 0$, inputs become perfectly homogeneous, implying then, in the limit, also that $q_{1}=q_{n}$. Consequently, condition (A.5) then holds indeed if $t<1 / 2$.

Proof of Proposition 2. The proof proceeds in analogy to that in Proposition 1, though now we can explicitly evaluate the derivative $d \pi_{v 1} /\left.d p^{1}\right|_{q 1}=0$ to see whether it is negative, which under linear demand would provide a sufficient and necessary condition for there to be no (full) foreclosure. Once $d \pi_{v I} /\left.d p^{1}\right|_{q^{1}=0}$ has been obtained, which is straightforward but not reported here for brevity's sake, one can show that the collected term relating to $\bar{c}$ is strictly negative, while the condition $d \pi_{v_{1}} /\left.d p^{1}\right|_{q^{1}=0}<0$ is easier to satisfy as products become more differentiated (lower $\theta$ ). In the limiting case with $\bar{c}=0$ and $\theta \rightarrow \infty$, the derivative becomes

$$
-\frac{(1-\beta)(N-f)\left[4 f^{2} N(1-\hat{\delta})+\hat{\delta}^{2} N^{2}(1+N+\hat{\delta} N)+\hat{\delta} N f(4+(4+3 \hat{\delta}) N)-4 f^{3}\right]}{\left[\hat{\delta} N(2+(2+\hat{\delta}) N)+2 f(4+(2+\hat{\delta}) N)-4 f^{2}\right] \cdot\left[\hat{\delta} N(1+(1+\hat{\delta}) N)+f(4+(2+3 \hat{\delta}) N)-2 f^{2}\right]},
$$

which is indeed strictly negative for all $1 \leqslant f<N$.

Proof of Proposition 4. As we take the limit $\theta \rightarrow \infty$, we have that all downstream products are offered at marginal cost of production. With vertical integration and the total output $q$, while thus $q_{n}=q / N$, we thus have the requirement that

$$
\bar{p}_{\emptyset}+\beta+\hat{\delta} \frac{q}{M}=p_{n}=1-q,
$$

such that, with $M=2$ and $\bar{p}=\bar{c}$, we have for the derived demand of firm $m=1$

$$
q^{1}=\frac{1}{2} \frac{1-\frac{1}{2}\left(p^{1}+\bar{c}\right)-\beta}{1+\hat{\delta} / 2}+\frac{1}{2 \hat{\delta}}\left(\bar{c}-p^{1}\right)
$$

which finally transforms to $q^{1}=(1 / \hat{\delta}(2+\hat{\delta}))\left[\hat{\delta}(1-\beta)+\bar{c}-p^{1}(1+\hat{\delta})\right]$. Pre-merger profits for $m=1$ are thus maximized at

$$
p^{1}=\frac{1}{2} \frac{\hat{\delta}(1-\beta)+\bar{c}}{1+\hat{\delta}} .
$$

At the other extreme, where $\theta=0$, we have for each product $p_{n}=1-N q_{n}$. Both the large firm, controlling $f$ products, and each small firm choose $p_{n}$ and $q_{\mathrm{n}}$ such that

$$
q_{n}=\frac{1}{N} \frac{1-\bar{p}_{\emptyset}-\beta}{2+\hat{\delta} / M} .
$$

For $m=2$ we then have the demand

$$
q^{1}=\frac{1-\frac{1}{2}\left(p^{1}+\bar{c}\right)-\beta}{2+\hat{\delta} / 2}+\frac{1}{2 \hat{\delta}}\left(\bar{c}-p^{1}\right),
$$

which transforms to $q^{1}=(1 / \hat{\delta}(4+\hat{\delta}))\left[\hat{\delta}(1-\beta)+2 \bar{c}-p^{1}(2+\hat{\delta})\right]$. Pre-merger profits for $m=1$ are thus maximized at

$$
p^{1}=\frac{1}{2} \frac{\hat{\delta}(1-\beta)+2 \bar{c}}{2+\hat{\delta}} \text {. }
$$

Comparing (A.6) with (A.7), we have that the pre-merger upstream margin is higher as $\theta \rightarrow \infty$ in case $1-\beta>\bar{c}$, which must hold to ensure that the second input is used.

Given symmetry for all products $n=1, \ldots . f$, the pre-merger margin of the large downstream firm is given by $p_{n}-C_{k}\left(q_{n}\right) / q_{n}$, where we can use $n=1$. Note now that as $\theta \rightarrow \infty, p_{n^{\prime}}$ is strictly lower and $q_{n^{\prime}}$ strictly higher for all other products $n^{\prime} \neq n$. Together with the obtained comparison for the input price $p^{1}$, it follows thus immediately that the pre-merger downstream margin is strictly lower for $\theta \rightarrow \infty$ than when $\theta=0$.

The assertion follows then from these observations together with Proposition 3.

\footnotetext{
goods.

${ }^{22}$ This includes intuitively the case of Cournot competition in differentiated products, where incentives to foreclose are lower than with homogeneous
} 


\section{References}

Akman, P., 2010. Consumer versus Customer: the devil in the detail. Journal of Law and Society 37, 315-344.

Avenel, E., Barlet, C., 2000. Vertical foreclosure, technological choice, and entry in the intermediate market. Journal of Economics and Management Strategy 9 , 211-230.

Bourreau, M., Hombert, J., Pouyet, J., Schutz, J., 2010. Upstream competition between vertically integrated firms. Journal of Industrial Economics, forthcoming.

Chen, Y., 2001. On vertical mergers and their competitive effect. Rand Journal of Economics 32, 667-685.

Choi, J.P., Yi, S.S., 2000. Vertical foreclosure with the choice of input specifications. Rand Journal of Economics 31, 717-743.

Church, J., 2004. The impact of vertical and conglomerate mergers on competition. Report prepared for the European Commission.

European Commission, 2007. Commission notice: guidelines on the assessment of non-horizontal mergers under the Council Regulation on the control of concentrations between undertakings, December 2007.

Gaudet, G., Van Long, N., 1996. Vertical integration, foreclosure, and profits with double marginalization. Journal of Economics and Management Strategy 5, 409-432.

Gilbert, R., Hastings, J., 2005. Vertical integration in gasoline supply: an empirical test of raising rivals' costs. Journal of Industrial Economics 53, 469-492.

Hart, O., Tirole, J., 1990. Vertical integration and market foreclosure. Brookings Papers on Economic Activity, $205-276$.

Higgins, R.S., 1999. Competitive vertical foreclosure. Managerial and Decision Economics 20, 229-237.

Höffler, F., Schmidt, K., 2008. Two tales on resale. International Journal of Industrial Organization 26, 1448-1460.

Inderst, R., Valletti, T., 2009. Indirect versus direct constraints in markets with vertical integration. Scandinavian Journal of Economics $111,527-546$.

Neven, D., Albaek, S., 2007. Economics at DG competition 2006-2007. Review of Industrial Organization 31, $139-153$.

Normann, H.-T., 2009. Vertical integration, raising rivals' costs and upstream collusion. European Economic Review 53, 461-480.

Ordover, J.A., Saloner, G., Salop, S.C., 1990. Equilibrium vertical foreclosure. American Economic Review 80, $127-142$.

Ordover, J., Shaffer, G., 2007. Wholesale access in multi-firm markets: when is it profitable to supply a competitor? International Journal of Industrial Organization 25, 1026-1045

Quirmbach, H.C., 1992. Sequential vertical integration. Quarterly Journal of Economics 107, 1101-1111.

Reiffen, D., 1992. Equilibrium vertical foreclosure: Comment. American Economic Review 82, 694-697.

Reiffen, D., Vita, M., 1995. Comment: is there new thinking on vertical mergers? Antitrust Law Journal 63, 917-941.

Reisinger, M., Schnitzer, M., 2009. Successive oligopolies with differentiated firms and endogenous entry. Mimeo.

Rey, P., Tirole, J., 2007. A primer on foreclosure. In: Armstrong, M., Porter, R. (Eds.), Handbook of Industrial Organization, vol. III. North-Holland, Amsterdam, pp. 2145-2220.

Salinger, M.A., 1988. Vertical mergers and market foreclosure. Quarterly Journal of Economics 103, 345-356.

Scheffman, D., Higgins, R., 2003. Twenty years of raising rivals' costs: history, assessment and future. George Mason Law Review 12 (2), $371-388$.

Schrader, A., Martin, S., 1998. Vertical market participation. Review of Industrial Organization 13, 321-331.

Shubik, M., Levitan, R., 1980. Market Structure and Behavior. Harvard University Press.

Warren-Boulton, F.R., 1974. Vertical control with variable proportions. Journal of Political Economy 82, 783-802.

Weyl, E.G., 2008. Pass-through as an economic tool. Mimeo. 